\title{
Extended Reynolds lubrication model for incompressible Newtonian fluid
}

\author{
Shintaro Takeuchi and Jingchen Gu \\ Department of Mechanical Engineering, Osaka University, \\ 2-1 Yamada-oka, Suita-city, Osaka 565-0871, Japan
}

(Received 12 March 2019; published 19 November 2019)

\begin{abstract}
An extended lubrication model is proposed for improvement of the lubrication theory by taking into account a larger surface-to-surface distance than that for the Reynolds lubrication theory. The analysis shows that when considering the non-negligible pressure gradient in the surface-normal direction, the local pressure is separated into (i) a base component satisfying the Reynolds lubrication theory and (ii) an adjusting component varying in the surface-normal direction, which is found to take the form proportional to the longitudinal derivative of the local velocity of the Couette-Poiseuille flow. Comparison of the results obtained by analytical and numerical methods for the lubrication between a moving curved object and stationary object shows that the proposed lubrication model reproduces the pressure distribution in both wall-normal and longitudinal directions. In a problem of a spherical particle approaching to a plane wall, the hydrodynamic force calculated by the proposed model exhibits an inverse-proportional trend to the surface-tosurface distance. The results suggest extended applicability of the lubrication theory to a non-Reynolds regime.
\end{abstract}

DOI: 10.1103/PhysRevFluids.4.114101

\section{INTRODUCTION}

Dense particulate flows containing large numbers of solid particles [1-5] are common in industrial applications and biological environments, and lubrication plays a crucial role in the interparticle region for determining the global behavior and dissipation of the particulate flows, including jamming transition via a lubrication breakdown [6-11]. During the simulation of the flow in the interparticle region on a fixed Cartesian grid, the surface distance of the interparticle gap (and/or narrow particle-wall gap) becomes comparable to or smaller than the grid size, and the fluid flow in that region is often unresolved. For a spherical two-particle system, the lubrication forces have been well described in the Stokes regime [12-16]. On the other hand, more general lubrication between objects of nonspherical geometries may be described by a small deformation approximation [17], the Benny-Lin equation for small perturbation in film flow on an inclined plane [18,19], or by the Reynolds lubrication equation [20] which shows that the pressure is independent of the wall-normal direction even for deformable particles such as biological cells [21,22] and bubbles or droplets [23].

Figure 1 shows a two-dimensional schematic of a narrow gap between two moving solid surfaces. The coordinate $y$ is in the surface-normal direction, and $x$ is taken to be normal to $y$. Here, $L$ and $H$ are the length scales in the longitudinal $(x)$ and surface-normal $(y)$ directions, respectively, and the

Published by the American Physical Society under the terms of the Creative Commons Attribution 4.0 International license. Further distribution of this work must maintain attribution to the author(s) and the published article's title, journal citation, and DOI. 


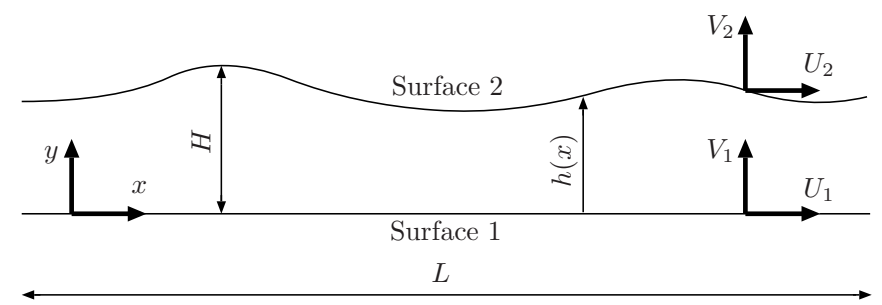

FIG. 1. Schematic of a narrow gap between two moving solid surfaces.

aspect ratio of the narrow gap is introduced as $\varepsilon=H / L$. The velocities in the $x$ and $y$ directions are introduced as $u$ and $v$, respectively, and the corresponding reference velocities are taken as $U$ and $V$. Then, the equation of continuity for an incompressible fluid,

$$
\frac{\partial u}{\partial x}+\frac{\partial v}{\partial y}=0
$$

gives the estimation $V=\varepsilon U$. The Reynolds lubrication theory [20] assumes a narrow gap of $\varepsilon \ll$ 1 , and the unsteady and convective terms are neglected for a small value of $\varepsilon^{2} \mathrm{Re}$, where $\mathrm{Re}=$ $\rho U L / \mu$ is the Reynolds number, and $\rho$ and $\mu$ are the density and viscosity of the fluid, respectively. Retaining only the $O\left[\varepsilon^{0}\right]$ terms, as explained later, the incompressible Navier-Stokes equations are reduced to the following equations:

$$
\frac{\partial p}{\partial x}=\mu \frac{\partial^{2} u}{\partial y^{2}}, \quad \frac{\partial p}{\partial y}=0,
$$

where $p$ is the pressure. Coupling this with Eq. (1) yields the Reynolds lubrication equation in which the dimension of the problem is reduced because the surface-normal variation of the pressure is eliminated, i.e., $p(x)$.

In our previous work [24], the Reynolds lubrication equation was incorporated into a particulate flow solver for correcting the flow in the interparticle region, and the numerical solution was confirmed to be in good agreement with the results of the analytical and independently conducted numerical studies (by direct numerical simulation) for eccentric-bearing film flow and fluid-particle interaction problems in a low Reynolds number range with less computational time. However, our previous study also revealed the limited applicability of the Reynolds lubrication equation to model particle-induced flows due to the strong constraints of $\varepsilon \ll 1$ and $\varepsilon^{2} \operatorname{Re} \ll 1$; only a limited configuration satisfies this condition, and in other cases, the variation in the wall-normal direction is non-negligible.

Indeed, in a relatively wide region of $\varepsilon \sim 1$, for example, the velocity components are comparable $(V \sim U)$. Further, the pressure gradient in the surface-normal direction may be non-negligible, and the Reynolds lubrication equation does not describe the correct film flow.

In the present study, we introduce a relaxed geometric condition of $\varepsilon \lesssim 1$ while $\varepsilon^{2}$ is still small enough (i.e., $\varepsilon^{2} \ll 1$ ), together with approximations of small gradient and curvature for surface profile. Then, an extended lubrication model is proposed by considering the non-negligible effect of the pressure variation in the surface-normal direction. To assess the validity of the extended lubrication model, the pressure fields obtained by the present model are compared with analytical and direct-numerical solutions for lubrication flows between moving and stationary walls.

\section{GOVERNING EQUATIONS}

The scaled variables $u_{*}=u / U, v_{*}=v / V, t_{*}=L / U, x_{*}=x / L, y_{*}=y / H$, and $p_{*}=p / P_{\text {ref }}$ are introduced in the incompressible Navier-Stokes equations, as well as the equation of continuity, Eq. (1), where $t$ is the time and $P_{\text {ref }}=\varepsilon^{-2} \mu U L^{-1}$. Then, the scaled Navier-Stokes equations are 
obtained as [25]

$$
\begin{aligned}
\varepsilon^{2} \operatorname{Re}\left(\frac{\partial u_{*}}{\partial t_{*}}+\boldsymbol{u}_{*} \cdot \nabla u_{*}\right) & =-\frac{\partial p_{*}}{\partial x_{*}}+\left(\varepsilon^{2} \frac{\partial^{2} u_{*}}{\partial x_{*}^{2}}+\frac{\partial^{2} u_{*}}{\partial y_{*}^{2}}\right), \\
\varepsilon^{4} \operatorname{Re}\left(\frac{\partial v_{*}}{\partial t_{*}}+\boldsymbol{u}_{*} \cdot \nabla v_{*}\right) & =-\frac{\partial p_{*}}{\partial y_{*}}+\varepsilon^{2}\left(\varepsilon^{2} \frac{\partial^{2} v_{*}}{\partial x_{*}^{2}}+\frac{\partial^{2} v_{*}}{\partial y_{*}^{2}}\right) .
\end{aligned}
$$

By eliminating all the terms smaller than the order of $\varepsilon^{2}$, the above equations are reduced to Eq. (2). On the other hand, assuming $\mathrm{Re} \ll 1$ and retaining the terms of $O\left[\varepsilon^{2}\right]$, we have the following set of equations:

$$
\begin{array}{ll}
\frac{\partial p_{*}}{\partial x_{*}}=\varepsilon^{2} \frac{\partial^{2} u_{*}}{\partial x_{*}^{2}}+\frac{\partial^{2} u_{*}}{\partial y_{*}^{2}}, \\
\frac{\partial p_{*}}{\partial y_{*}}= \\
\varepsilon^{2} \frac{\partial^{2} v_{*}}{\partial y_{*}^{2}} .
\end{array}
$$

The first term in the right-hand side of Eq. (3a) is reevaluated as follows:

$$
\varepsilon^{2} \frac{\partial^{2} u_{*}}{\partial x_{*}^{2}}=\varepsilon^{2}\left[\frac{\partial^{2} u_{*}}{\partial h_{*}^{2}}\left(\frac{d h_{*}}{d x_{*}}\right)^{2}+\frac{\partial u_{*}}{\partial h_{*}} \frac{d^{2} h_{*}}{d x_{*}^{2}}\right],
$$

where $h_{*}=h / H$. This equation suggests that the term $\partial^{2} u / \partial x^{2}$ is smaller than the order of $\varepsilon^{2}$ when both the gradient and curvature of the surface profile remain small. Equation (4) may be represented in an alternative form: assuming that the gap width $h(x)$ changes only slightly such that the variation of the width, $\Delta h$, is much smaller than $H$, then

$$
\varepsilon^{2} \frac{\partial^{2} u_{*}}{\partial x_{*}^{2}}=\frac{H^{2}}{U} \frac{\partial^{2} u}{\partial x^{2}}=\frac{H^{2}}{U}\left[\frac{\partial^{2} u}{\partial h^{2}}\left(\frac{d h}{d x}\right)^{2}+\frac{\partial u}{\partial h} \frac{d^{2} h}{d x^{2}}\right] \simeq \varepsilon^{2}\left(\frac{\partial^{2} u_{*}}{\partial h_{*}^{2}} \frac{\Delta h^{2}}{H^{2}}+\frac{\partial u_{*}}{\partial h_{*}} \frac{\Delta h}{H}\right),
$$

suggesting that the condition $\Delta h / H \ll 1$ enables elimination of the second-order $x$ derivative of $u$. Therefore, in the present study, we employ the following set of equations for lubrication:

$$
\begin{aligned}
& \frac{\partial p}{\partial x}=\mu \frac{\partial^{2} u}{\partial y^{2}}, \\
& \frac{\partial p}{\partial y}=\mu \frac{\partial^{2} v}{\partial y^{2}}
\end{aligned}
$$

in a relatively wide region (i.e., non-Reynolds lubrication condition) of $\varepsilon \lesssim 1, \varepsilon^{2} \ll 1$, and $\Delta h / H \ll 1$.

\section{MODELING}

By solving Eqs. (1) and (6), a lubrication model is constructed to describe the pressure variations in both the longitudinal and surface-normal directions. The following two lemmas are introduced.

Lemma 1. The local pressure gradient in the $x$ direction, $\partial p / \partial x$, is regarded as a function of $x$ (and is negligibly relevant to $y$ ).

Lemma 2. The pressure can be separated into the base and adjusting components:

$$
p(x, y)=p_{\mathrm{b}}(x)+p_{\text {adj }}(x, y),
$$

where no more independent functions of $x$ are separable from $p_{\text {adj }}$.

The proofs of the lemmas are given in Appendix A.

By Lemma 1, we obtain the following form of $u$ by integrating Eq. (6a) twice with respect to $y$ :

$$
u=\frac{1}{\mu} \frac{\partial p}{\partial x} \frac{y^{2}}{2}+f_{1}(x) y+f_{2}(x)
$$


where $f_{1}$ and $f_{2}$ are the integral constants. The boundary conditions, $u=U_{1}$ at $y=0$ (surface 1) and $u=U_{2}$ at $y=h(x)$ (surface 2), identify these constants as

$$
f_{1}(x)=\frac{U_{\mathrm{r}}}{h}-\frac{h}{2 \mu} \frac{\partial p}{\partial x}, \quad f_{2}(x)=U_{1},
$$

where $U_{\mathrm{r}}=U_{2}-U_{1}$. From $\partial v / \partial y=-\partial u / \partial x$ and Eq. (8),

$$
v=-\frac{1}{\mu} \frac{\partial^{2} p}{\partial x^{2}} \frac{y^{3}}{6}-\frac{d f_{1}}{d x} \frac{y^{2}}{2}-\frac{d f_{2}}{d x} y+V_{1}
$$

is obtained, where one of the boundary conditions $\left.v\right|_{y=0}=V_{1}$ is used. The other boundary condition, $\left.v\right|_{y=h}=V_{2}$, imposes the following relation:

$$
\frac{d f_{2}}{d x}=-\frac{V_{\mathrm{r}}}{h}-\frac{h^{2}}{6 \mu} \frac{\partial^{2} p}{\partial x^{2}}-\frac{h}{2} \frac{d f_{1}}{d x}
$$

where $V_{\mathrm{r}}=V_{2}-V_{1}$. Substituting Eq. (9) into the above equation, an equation analogous to the Reynolds lubrication equation is derived:

$$
\frac{h^{3}}{12 \mu} \frac{\partial^{2} p}{\partial x^{2}}+\frac{h^{2}}{4 \mu} \frac{d h}{d x} \frac{\partial p}{\partial x}=\frac{h}{2} \frac{d U_{\mathrm{r}}}{d x}-\frac{U_{\mathrm{r}}}{2} \frac{d h}{d x}+h \frac{d U_{1}}{d x}+V_{\mathrm{r}}
$$

Equation (11) suggests that, although it considers the pressure variation in the surface-normal direction, the surface pressure still obeys the Reynolds lubrication equation. Considering Lemma 1 , this can be written as follows:

$$
\frac{\partial p}{\partial x} \simeq \frac{d p_{\mathrm{Re}}}{d x} \quad \text { and } \quad \frac{\partial^{2} p}{\partial x^{2}} \simeq \frac{d^{2} p_{\mathrm{Re}}}{d x^{2}}
$$

where $p_{\operatorname{Re}}(x)$ is the pressure determined by the Reynolds lubrication equation.

An alternative form of $v$ is derived to obtain a closed system of $p$. By integrating Eq. (6b) with respect to $y$ and using Lemma $2, \partial v / \partial y$ is related to $p_{\text {adj }}$ as

$$
\begin{aligned}
p(x, y) & =f_{3}(x)+\mu \frac{\partial v}{\partial y}=p_{\mathrm{b}}(x)+p_{\text {adj }}(x, y), \\
\therefore \quad \frac{\partial v}{\partial y} & =\frac{p_{\mathrm{b}}(x)-f_{3}(x)}{\mu}+\frac{p_{\mathrm{adj}}(x, y)}{\mu},
\end{aligned}
$$

where $f_{3}$ is an integral constant. Comparing the above equation with the $y$-dependent terms in $-\partial u / \partial x$, the following relation is obtained:

$$
-\frac{\partial}{\partial x}\left[\frac{1}{\mu} \frac{\partial p}{\partial x} \frac{y^{2}}{2}+f_{1}(x) y\right]=\frac{p_{\mathrm{adj}}}{\mu} .
$$

From the above equation, the order of magnitude of $\partial p_{\text {adj }} / \partial x$ is found to be $\mu U L^{-2}$, while those of $\partial p / \partial x$ and $d p_{\operatorname{Re}} / d x$ are $\varepsilon^{-2} \mu U L^{-2}\left(=P_{\text {ref }} / L\right)$, which reads $p_{\mathrm{b}} \simeq p_{\operatorname{Re}}$ from Eq. (7) provided $\varepsilon^{2} \ll 1$. On the other hand, $\partial p_{\mathrm{b}} / \partial y=0$ and Eq. (6b) yield

$$
O\left[\frac{\partial p}{\partial y}\right]=O\left[\frac{\partial p_{\text {adj }}}{\partial y}\right]=\frac{1}{\varepsilon^{2}} \frac{\mu V}{L^{2}} .
$$

This fact characterizes the present problem; although $p_{\text {adj }}$ is small in comparison to $p_{\text {Re }}, \partial p_{\text {adj }} / \partial y$ exhibits a comparable order of magnitude to $\partial p_{\operatorname{Re}} / \partial x$. Equation (15) may seem to be inconsistent with the $P_{\text {ref }}$-based evaluation: recalling $O[p]=O\left[p_{\text {Re }}\right]=P_{\text {ref }}$, then $O[\partial p / \partial y]=O\left[\left(P_{\text {ref }} / H\right) \partial p_{*} / \partial y_{*}\right]=$ $\varepsilon^{-4} \mu V L^{-2}$. However, $p_{\text {Re }}$ (i.e., the major term in $p$ ) is independent of $y$, and therefore, the $P_{\text {ref }}$-based estimation is impractical for evaluating $\partial p / \partial y$. Instead, the $y$ gradient of $p_{\text {adj }}$ (as part of $p$ ) should be balanced in magnitude with the viscous term on the right-hand side of Eq. (6b), as shown in Eq. (15). 


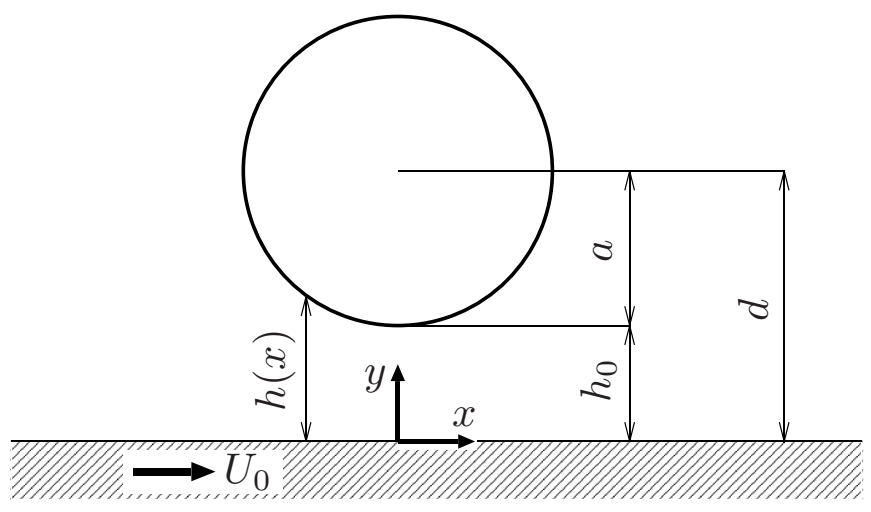

FIG. 2. Schematic of a fixed cylinder above a moving plate.

By replacing $p_{\text {adj }}$ with $p-p_{\text {Re }}$, Eq. (14) is transformed into a closed equation of $p(x, y)$ :

$$
\mu \frac{\partial}{\partial x}\left[-\frac{y(h-y)}{2 \mu} \frac{\partial p}{\partial x}\right]+p=p_{\mathrm{Re}}-\mu y \frac{d\left(U_{\mathrm{r}} / h\right)}{d x} .
$$

The crucial advantage of this equation is that, similar to the Reynolds lubrication equation, $\partial / \partial y$ does not appear: given $p_{\text {Re }}$, Eq. (16) is solved for a fixed $y$. Furthermore, a completely closed form of the equation for $p$ is obtained by eliminating $p_{\operatorname{Re}}$ with the Reynolds lubrication equation (see Appendix B). However, the Reynolds lubrication equation and Eq. (16) are pseudo-Poisson and Helmholtz types, respectively, and the numerical solutions of those elliptic-type equations are computationally intensive.

On the other hand, Eq. (14) also indicates a simpler procedure. Substitution of Eq. (12) into (14) identifies $p_{\text {adj }}$ as

$$
p_{\text {adj }} \simeq-\mu \frac{\partial}{\partial x}\left[-\frac{y(h-y)}{2 \mu} \frac{d p_{\mathrm{Re}}}{d x}+\frac{y}{h} U_{\mathrm{r}}\right] .
$$

In this equation, the terms inside the $x$ derivative represent the velocity of the Couette-Poiseuille flow. Equation (17) suggests that $p_{\text {adj }}$ is the pressure adjustment due to the spatial change of the local Couette-Poiseuille flow, induced by the gradient of $p_{R e}$ and moving walls, in a narrow gap. Therefore, in a feasible extended lubrication model for the non-Reynolds regime, $p_{\operatorname{Re}}$ is obtained from the Reynolds lubrication equation, and then, with $p_{\text {adj }}(x, y)$ determined by Eq. (17), the pressure is eventually given by

$$
p(x, y)=p_{\mathrm{Re}}(x)+p_{\text {adj }}(x, y) .
$$

The three-dimensional version of the extended lubrication model is presented in Appendix C.

\section{VALIDATION}

To assess the validity of the proposed extended lubrication model, pressure fields in the small gaps are compared with analytical and numerical solutions.

Wannier [26] demonstrated the analytical solution of the Stokes equation for the flow between a flat moving plate and a cylinder (as schematically shown in Fig. 2), and the pressure is given as

$$
\frac{p_{\mathrm{Wa}}(x, y)}{\mu}=-\frac{B x(s+y)}{y^{2}(\delta+s)^{2}}-\frac{C x(s-y)}{y^{2}(\delta-s)^{2}}-\frac{4 F s x}{y\left(\delta^{2}-s^{2}\right)},
$$




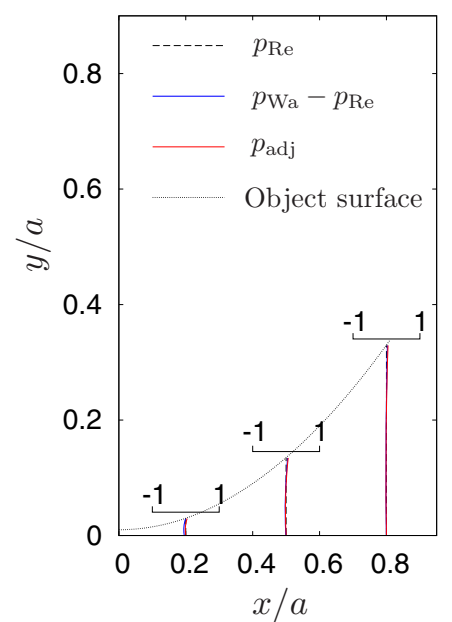

(a)

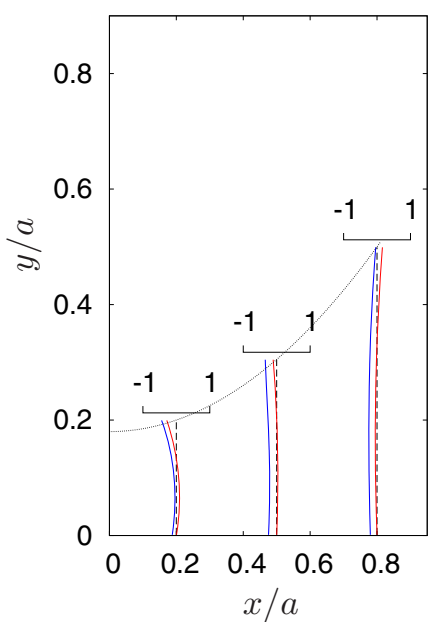

(b)

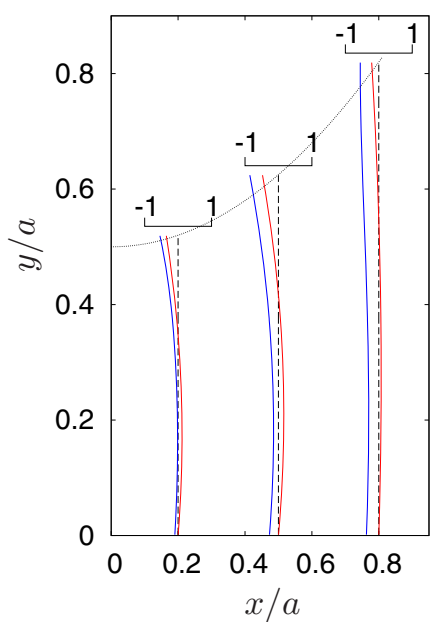

(c)

FIG. 3. Comparison of the cross-channel profiles of the pressures obtained by the present model [Eq. (18)] and the Wannier solution [Eq. (19)] plotted relative to $p_{\operatorname{Re}}$ [Eq. (20)] at three longitudinal positions $(x / a=$ $0.2,0.5$, and 0.8 ) for three different cylinder locations (a) $d / a=1.01$, (b) 1.18 , and (c) 1.5 . The pressure value is normalized by $\mu U_{0} / h_{0}$.

where

$$
\begin{aligned}
s^{2} & =d^{2}-a^{2}, \\
\delta & =\left(x^{2}+y^{2}+s^{2}\right) / 2 y, \\
B & =2(d+s) U_{0} / \ln \Gamma, \\
C & =2(d-s) U_{0} / \ln \Gamma, \\
F & =U_{0} / \ln \Gamma, \\
\Gamma & =(d+s) /(d-s) .
\end{aligned}
$$

On the other hand, the Reynolds lubrication equation gives the pressure for this flow as

$$
p_{\operatorname{Re}}(x)=-\frac{8 a^{2} \mu U_{0} x}{\left(2 a h_{0}+x^{2}\right)^{2}},
$$

where the surface-to-surface distance is approximated as $h \simeq h_{0}+x^{2} / 2 a$, and the boundary pressures are assigned as $p_{\operatorname{Re}}( \pm \infty)=0$.

Figure 3 compares the cross-channel profiles of the relative pressures to $p_{\operatorname{Re}}$ obtained by the extended lubrication model and the Wannier solution. The pressure is normalized by $\mu U_{0} / h_{0}$. The profiles are shown at three different $x$ positions $(x / a=0.2,0.5$, and 0.8$)$ for three different cylinder levels $d / a=1.01,1.18$, and 1.50. Those $d / a$ values correspond to $\varepsilon=h_{0} / 2 a=0.005,0.09$, and 0.25 , and $\Delta h / H=a / d=0.99,0.84$, and 0.67, respectively. Figure 3(a) shows that the pressure for the case of $d / a=1.01$ is well described by the Reynolds lubrication equation. For the case of $d / a=1.18$, on the other hand, Fig. 3(b) suggests that the present extended lubrication model $\left(p_{\text {adj }}\right)$ reasonably captures the trend of $p_{\mathrm{Wa}}-p_{\mathrm{Re}}$ at $x / a=0.2$, where the locally evaluated value $\varepsilon=h(0.2 a) / 2 a \fallingdotseq 0.1$ may be around an upper limit for $\varepsilon^{2} \ll 1$ and $\Delta h / H \simeq \Delta h /\left.h\right|_{x=0.2 a} \fallingdotseq$ $0.02 a / 0.2 a=0.1$ is also reasonable. The deviation of the wall-normal distribution of $p_{\text {adj }}$ from the Wannier case becomes non-negligible at $x / a=0.4$ and 0.8 ; in particular, the deviation at $x / a=0.4$ is large as the sign of $\partial^{2} p_{\text {adj }} / \partial y^{2}$ changes (i.e., convex to concave profiles along the $y$ axis) at around $x / a=0.5$, as typically observed later in a pressure contour map (Fig. 4). The case of $d / a=1.5$ 

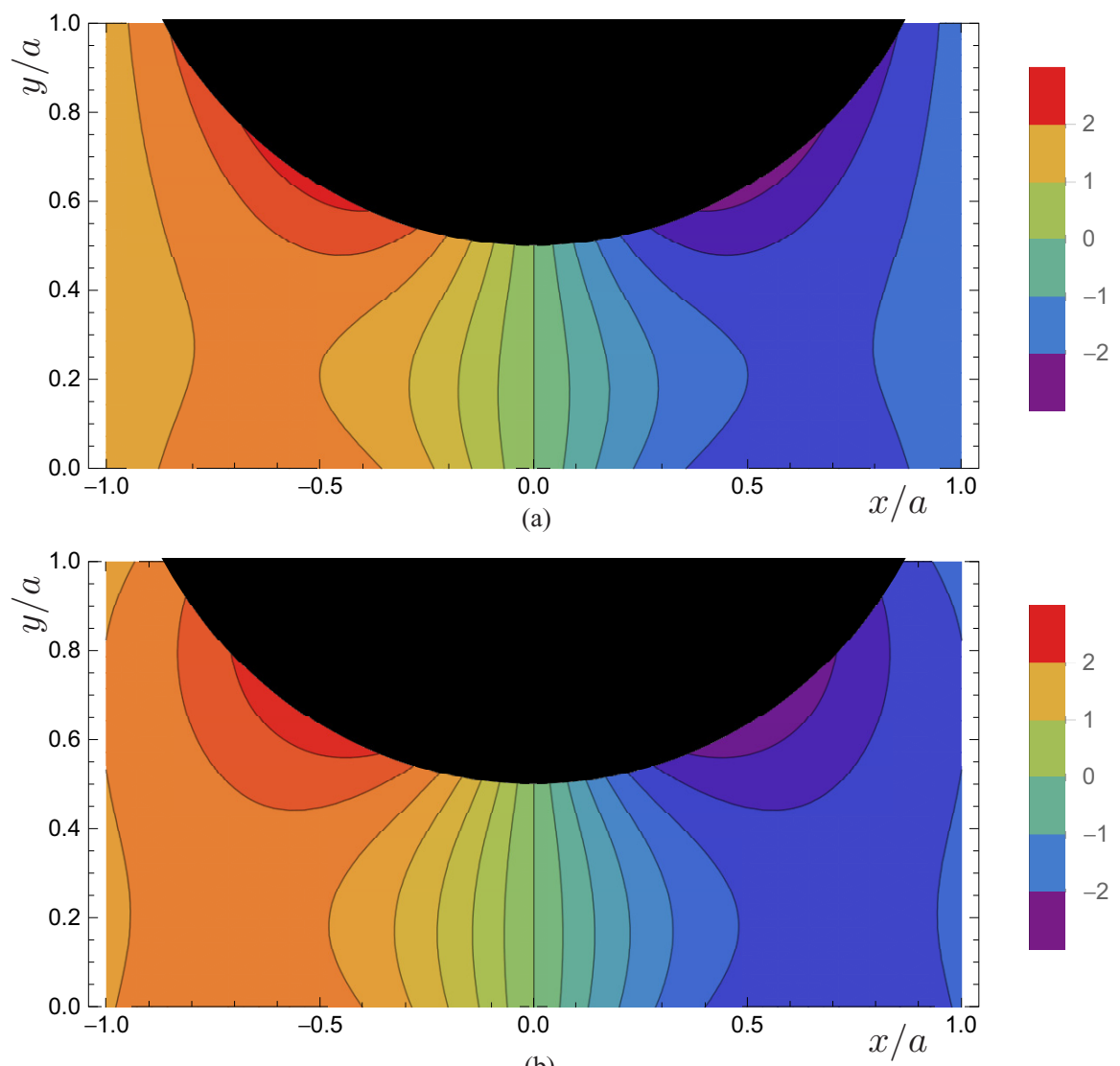

(b)

FIG. 4. Comparison of the pressures obtained by Eqs. (18) and (19) for $d / a=1.5$. (a) Extended lubrication model, Eq. (18). (b) Wannier solution [27], Eq. (19).

( $\left.\varepsilon^{2}=0.125\right)$ may be even farther from the condition of $\varepsilon^{2} \ll 1$, and the $\Delta h / H$ value is not fully ideal to justify Eq. (6b) for the entire $x$ range. However, the local value $\Delta h /\left.h\right|_{x=0.2 a} \fallingdotseq 0.02$ suggests the applicability to a limited $x$ range, and, as Fig. 3(c) shows, the extended lubrication model still works to improve towards the correct pressure distribution. Figure 4 shows the comparison of the pressure distributions obtained by Eqs. (18) and (19) for $d / a=1.5$. Considering that $p_{\operatorname{Re}}$ is only $x$ dependent, the effect of the adjustment by $p_{\text {adj }}$ is remarkable. Both Figs. 3 and 4 suggest that the adjustment $\left(p_{\text {adj }}\right.$ ) added to $p_{\text {Re }}$ improves the $y$-dependent trends of the pressure towards the Wannier flow even in a wide gap region.

For a second validation case, the flow induced by a curved object traveling at a constant speed is set up to highlight the noncylindrical geometric effect and is compared with the numerical solution. As schematically shown in Fig. 5, a corrugated plate of sinusoidal geometry is placed in the middle $y$ level of the domain bounded by the stationary flat solid walls, and the object is towed at a constant speed of $U_{0}$ in the positive $x$ direction:

$$
h(x, t)=H_{0}\left(1+\delta \cos \left[k\left(x-U_{0} t\right)\right]\right),
$$

where $H_{0}$ is the half-channel height and $\delta$ is the nondimensional parameter between 0 and 1 . The wave number $k$ is set to $2 \pi / L_{0}$. The periodic boundary condition is applied in the $x$ direction, and the no-slip condition is imposed on the solid surfaces. Following the lubrication analysis in Ref. [27], 


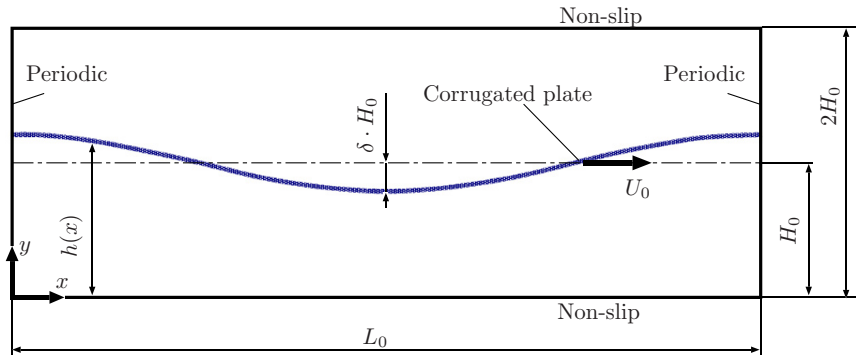

FIG. 5. Schematic of a moving corrugated plate in a parallel channel.

the $p_{\mathrm{Re}}$ for this problem is given as follows:

$$
p_{\operatorname{Re}}(x, t)=-6 \mu U_{0} \frac{h(x, t)+H_{0}}{\left(k H_{0}\right)^{2}\left(2+\delta^{2}\right)} \frac{\partial h^{-1}(x, t)}{\partial x} .
$$

For comparison, a direct numerical simulation (DNS) is carried out with a fully validated method [27]. The spatial resolution is fixed as $\Delta / H_{0}=0.05$ in both directions, where $\Delta$ is the grid spacing. The Reynolds number for the DNS is set as $\operatorname{Re}=\rho U_{0} L_{0} / \mu=1$.

Figure 6 compares the instantaneous $\left(t=4 \pi / k U_{0}\right)$ pressure profiles along the line $y=H_{0}(1-$ $\delta) / 2$ (i.e., the midpoint of the narrowest channel width) obtained by the extended lubrication model and the DNS for different aspect ratios and amplitude parameters in the following ranges: $H_{0} / L_{0}=$ $0.1,0.5$ and $\delta=0.1,0.25$. In the case of the smaller aspect ratio $\left(H_{0} / L_{0}=0.1\right)$, the predictions of the extended lubrication model are close to the DNS for both $\delta$ cases. Note that the corresponding values of $\varepsilon^{2}\left[=\left(\max h / L_{0}\right)^{2}\right]$ are 0.012 and 0.015 for $\delta=0.1$ and 0.25 , respectively, which are sufficiently small, whereas in the case of the larger aspect ratio 0.5 (correspondingly $\varepsilon^{2}=0.30$ ), the pressure exhibits large deviation as observed from the figure. On the other hand, the effect of $\Delta h / H$ is relatively small for this periodic geometry of the gap because, with $\Delta h$ and $H$ evaluated as $2 \delta H_{0}$ and $H_{0}(1+\delta), \Delta h / H$ is about the same order of magnitude as $\delta$. Therefore, the comparison with the DNS result shows that the proposed extended lubrication model reasonably predicts the pressure distribution of the film flow in the noncircular gap when $\varepsilon^{2}$ is much smaller than unity.

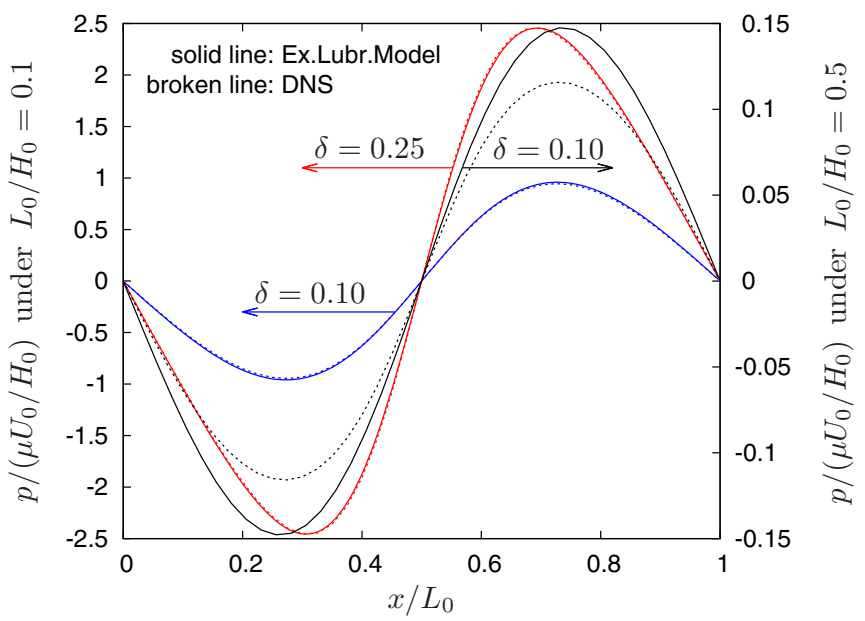

FIG. 6. Comparison of the pressure distributions along $y=\left(H_{0}-\delta H_{0}\right) / 2$ obtained by the extended lubrication model (solid lines) and by DNS (broken lines) for different values of $\delta$ and aspect ratio. Ex. Lubr. Model denotes Eq. (18) with $p_{\text {Re }}$ given by Eq. (22). 


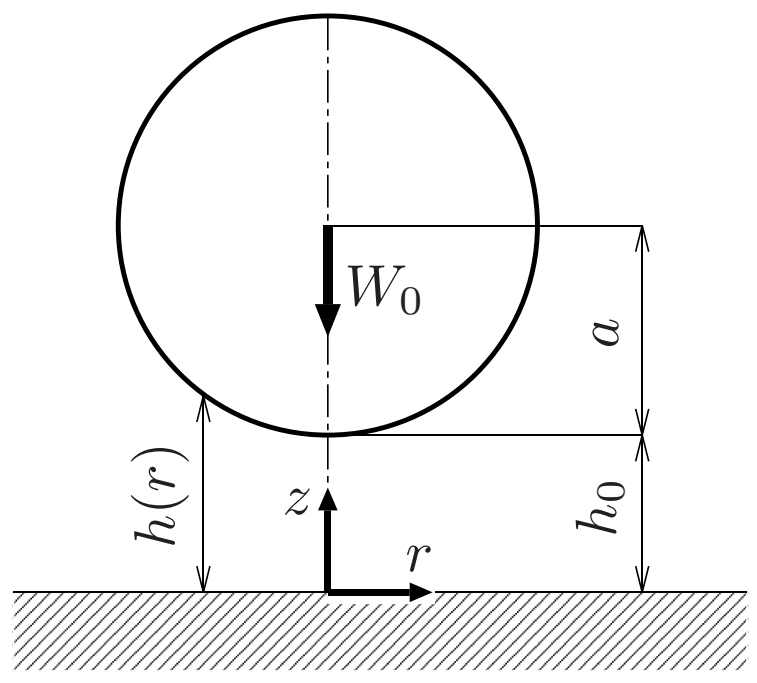

FIG. 7. Schematic of a spherical particle approaching to a flat wall.

The third validation case demonstrates the hydrodynamic force on the center of a spherical particle generated by the lubrication in the region between a plane wall and the particle approaching at a constant speed with no angular velocity, as illustrated in Fig. 7. The pressure that satisfies the Reynolds lubrication equation is given as [28]

$$
p_{\operatorname{Re}}(r)=\frac{3 \mu a W_{0}}{\left(h_{0}+r^{2} / 2 a\right)^{2}},
$$

where the paraboloid approximation $h(r) \simeq h_{0}+r^{2} / 2 a$ is assumed for the gap distance. For the present axisymmetric problem, the adjusting component of the pressure [Eq. (C1) in Appendix C] is simplified to the following form:

$$
p_{\text {adj }}=-\mu \frac{\partial}{\partial r}\left[-\frac{z[h(r)-z]}{2 \mu} \frac{d p_{\mathrm{Re}}}{d r}\right],
$$

where $z$ represents the distance in the wall-normal direction. With the above approximation for the profile $h(r)$, the forces at the particle center are calculated as follows:

$$
\begin{aligned}
\int_{0}^{a} p_{\operatorname{Re}} 2 \pi r d r & =\frac{6 \pi \mu a W_{0}}{\varepsilon}\left(1-\frac{2 \varepsilon}{1+2 \varepsilon}\right), \\
\int_{0}^{a}\left(p_{\operatorname{Re}}+\left.p_{\mathrm{adj}}\right|_{z=h}\right) 2 \pi r d r & =\frac{6 \pi \mu a W_{0}}{\varepsilon}\left(1+2 \varepsilon \ln \frac{2 \varepsilon}{1+2 \varepsilon}\right),
\end{aligned}
$$

where $\varepsilon=h_{0} / a$. Both cases indicate that the major term is $\varepsilon^{-1}$ in the limit of $\varepsilon \rightarrow 0$, which is consistent with the prediction by asymptotic analysis [12].

\section{CONCLUDING REMARKS}

Our extended lubrication model proposed for a relatively wide range of aspect ratios $\varepsilon$ satisfying $\varepsilon \lesssim 1$ and $\varepsilon^{2} \ll 1$ enables decomposition of the pressure into two components: the contribution by the Reynolds lubrication theory $\left(p_{\mathrm{Re}}\right)$ and the adjusting component $\left(p_{\text {adj }}\right)$ varying in both longitudinal and wall-normal directions. Although the contribution of the latter is small in magnitude, its gradient in the surface-normal direction is significant. The adjusting component of pressure was described with the longitudinal derivative of the local velocity of the Couette-Poiseuille flow 
driven by $p_{\mathrm{Re}}$ and the tangential velocity of the walls. Through comparison with the analytical and numerical results, the extended lubrication model successfully predicted the pressure distribution, particularly the surface-normal distribution, and the force acting on the object center, regardless of the geometry (even for noncircular/nonspherical) of the object.

This method is easily applicable for analyzing film flow in an interparticle narrow gap; by solving the Reynolds lubrication equation with a combined Eulerian-Lagrangian formulation as demonstrated in Ref. [24], the efficient solution of a film flow under an unresolved situation is facilitated by the extended lubrication model presented herein. In future works, a method for modeling the film flow in the network of the narrow gaps between the particles will be developed to study dense particulate flows.

\section{ACKNOWLEDGMENTS}

The authors thank Dr. R. Onishi (Japan Agency for Marine-Earth Science and Technology, JAMSTEC), Dr. T. Fukada (Central Research Institute of Electric Power Industry, CRIEPI), and Professor T. Kajishima (Osaka University) for fruitful discussion. J.C.G. gratefully acknowledges the financial support by AdvanceSoft Corporation, Tokyo, Japan. This work is partly supported by Grants-in-Aid (B) No. 16H04271 and No. 17H03174 of the Japan Society for the Promotion of Science (JSPS).

\section{APPENDIX A: PROOFS OF LEMMAS 1 AND 2}

Lemma 1. The pressure gradient in the $x$ direction $\partial p / \partial x$ is a function of $x$ and is irrelevant to $y$ in the relatively wide region of $\varepsilon \lesssim 1$ and $\varepsilon^{2} \ll 1$.

Proof. The governing equations are Eqs. (1) and (6). Integrating Eq. (6b) with respect to $y$,

$$
\frac{\partial v}{\partial y}=\frac{1}{\mu} p(x, y)+g(x),
$$

where $g(x)$ is an integral constant. Taking the derivative of Eq. (A1) with respect to $x$,

$$
\frac{\partial^{2} v}{\partial x \partial y}=\frac{1}{\mu} \frac{\partial p}{\partial x}+\frac{d g}{d x} .
$$

According to Eq. (1),

$$
\frac{\partial^{2} u}{\partial x^{2}}=-\frac{\partial^{2} v}{\partial x \partial y}=-\frac{1}{\mu} \frac{\partial p}{\partial x}-\frac{d g}{d x},
$$

and substituting Eq. (6a) into the above equation, we obtain

$$
\frac{\partial^{2} u}{\partial x^{2}}+\frac{\partial^{2} u}{\partial y^{2}}=-\frac{d g}{d x} .
$$

Considering the relatively wide region of $\varepsilon^{2} \ll 1$, we can reasonably say that

$$
\frac{\partial^{2} u}{\partial x^{2}}=o\left(\frac{\partial^{2} u}{\partial y^{2}}\right)
$$

where the Landau symbol $o(X)$ means the high order of $X$. Equation (A4) suggests that $\partial^{2} u / \partial y^{2}$ is treated as a function of $x$, and therefore, $\partial p / \partial x$ is also a function of $x$ from Eq. (6a).

Lemma 2. The pressure $p(x, y)$ can be separated into $p(x, y)=p_{\mathrm{b}}(x)+p_{\text {adj }}(x, y)$, where no more independent functions of $x$ are separable from $p_{\text {adj }}$. 
Proof. Integrating Eq. (6a) twice with respect to $y$ and using Lemma 1, $u$ takes the following form:

$$
u=\frac{1}{\mu} \frac{\partial p}{\partial x} \frac{y^{2}}{2}+f_{1}(x) y+f_{2}(x)
$$

where $f_{1}$ and $f_{2}$ are the integral constants. According to Eq. (1), $\partial v / \partial y$ is also a second-order polynomial of $y$ :

$$
\frac{\partial v}{\partial y}=a(x) y^{2}+b(x) y+c(x)
$$

where $a, b$, and $c$ are the coefficients determined by the boundary condition on the solid surfaces. On the other hand, integrating Eq. (6b) with respect to $y, p$ takes the following form:

$$
\begin{aligned}
p & =\mu \frac{\partial v}{\partial y}-\mu g(x) \\
& =\mu[c(x)-g(x)]+\mu\left[a(x) y^{2}+b(x) y\right] .
\end{aligned}
$$

As a result, the pressure $p(x, y)$ can be separated into $p(x, y)=p_{\mathrm{b}}(x)+p_{\text {adj }}(x, y)$.

\section{APPENDIX B: EXTENDED LUBRICATION EQUATION}

A closed form of the equation for pressure $p(x, y)$ is obtained by substituting $p_{\text {Re }}$ of Eq. (16) into the Reynolds lubrication equation

$$
\frac{d}{d x}\left[\frac{h^{3}}{12 \mu} \frac{d p_{\mathrm{Re}}}{d x}\right]=\frac{h^{2}}{2} \frac{d}{d x}\left(\frac{U_{\mathrm{r}}}{h}\right)+h \frac{d U_{1}}{d x}+V_{r}
$$

as

$$
\frac{\partial}{\partial x}\left\{\frac{h^{3}}{12} \frac{\partial^{2}}{\partial x^{2}}\left[-\frac{y(h-y)}{2 \mu} \frac{\partial p}{\partial x}+\frac{y}{h} U_{\mathrm{r}}\right]+\frac{h^{3}}{12 \mu} \frac{\partial p}{\partial x}\right\}=\frac{h^{2}}{2} \frac{d}{d x}\left(\frac{U_{\mathrm{r}}}{h}\right)+h \frac{d U_{1}}{d x}+V_{r} .
$$

This form of equation may be useful when handling the governing equation of $p$ [not in a separate form with $p_{\text {Re }}$ and $p_{\text {adj }}$ as in Eq. (18)] for solving a coupled system with object motion.

\section{APPENDIX C: THREE-DIMENSIONAL MODEL}

The three-dimensional version of the extended lubrication model is realized by a procedure similar to that applicable for two dimensions (Sec. III). For a surface $z=h(x, y)$ traveling at the relative velocity $\left(U_{\mathrm{r}}, V_{\mathrm{r}}, W_{\mathrm{r}}\right)$ in the $(x, y, z)$ direction with respect to the bottom-wall velocity $\left(U_{1}, V_{1}, W_{1}\right)$, the result is given as follows:

$$
\begin{aligned}
& p(x, y, z)=p_{\text {Re }}(x, y)+p_{\text {adj }}(x, y, z) \\
& p_{\text {adj }}=-\mu \nabla_{2} \cdot\left[-\frac{z(h-z)}{2 \mu} \nabla_{2} p_{\operatorname{Re}}+\frac{z}{h}\left(\begin{array}{c}
U_{\mathrm{r}} \\
V_{\mathrm{r}}
\end{array}\right)\right]
\end{aligned}
$$

Here, $\nabla_{2}=(\partial / \partial x, \partial / \partial y)$, and $p_{\mathrm{Re}}$ is the solution of the following Reynolds lubrication equation:

$$
\nabla_{2} \cdot\left(\frac{h^{3}}{12 \mu} \nabla_{2} p_{\text {Re }}\right)=\frac{h^{2}}{2} \nabla_{2} \cdot\left(\begin{array}{l}
U_{\mathrm{r}} / h \\
V_{\mathrm{r}} / h
\end{array}\right)+h \nabla_{2} \cdot\left(\begin{array}{l}
U_{1} \\
V_{1}
\end{array}\right)+W_{\mathrm{r}} .
$$


The velocity components $(u, v, w)$ in the $(x, y, z)$ directions are given as follows:

$$
\begin{aligned}
\left(\begin{array}{l}
u \\
v
\end{array}\right) & =-\frac{\nabla_{2} p}{2 \mu} z(h-z)+\left(\begin{array}{c}
z U_{\mathrm{r}} / h+U_{1} \\
z V_{\mathrm{r}} / h+V_{1}
\end{array}\right), \\
w & =-\frac{z^{2}}{12 \mu} \nabla_{2} \cdot\left[(2 z-3 h) \nabla_{2} p\right]-\frac{z}{2} \nabla_{2} \cdot\left(\begin{array}{c}
z U_{\mathrm{r}} / h+2 U_{1} \\
z V_{\mathrm{r}} / h+2 V_{1}
\end{array}\right)+W_{1} .
\end{aligned}
$$

[1] J. M. Nouri, J. H. Whitelaw, and M. Yianneskis, Particle motion and turbulence in dense two-phase flows, Int. J. Multiphase Flow 13, 729 (1987).

[2] H. H. Hu, Direct simulation of flows of solid-liquid mixtures, Int. J. Multiphase Flow 22, 335 (1996).

[3] T. Ingrid and G. Marte, Fluid lubrication effects on particle flow and transport in a channel, Int. J. Multiphase Flow, 65, 143 (2014).

[4] S. Bogner, S. Mohanty, and U. Rude, Drag correlation for dilute and moderately dense fluid-particle systems using the lattice Boltzmann method, Int. J. Multiphase Flow 68, 71 (2015).

[5] J. Gu, S. Takeuchi, T. Fukada, and T. Kajishima, Vortical flow patterns by the cooperative effect of convective and conductive heat transfers in particle-dispersed natural convection, Int. J. Heat Mass Transf. 130, 946 (2019).

[6] J. R. Melrose, J. H. van Vliet, and R. C. Ball, Continuous Shear Thickening and Colloid Surfaces, Phys. Rev. Lett. 77, 4660 (1996).

[7] J. R. Melrose and R. C. Ball, Continuous shear thickening transitions in model concentrated colloids-The role of interparticle forces, J. Rheol. 48, 937 (2004).

[8] R. Seto, R. Mari, J. F. Morris, and M. M. Denn, Discontinuous Shear Thickening of Frictional HardSphere Suspensions, Phys. Rev. Lett. 111, 218301 (2013).

[9] R. Mari, R. Seto, J. F. Morris, and M. M. Denn, Shear thickening, frictionless and frictional rheologies in non-Brownian suspensions, J. Rheol. 58, 1693 (2014).

[10] C. Ness and J. Sun, Shear thickening regimes of dense non-Brownian suspensions, Soft Matter 12, 914 (2016).

[11] C. Ness and J. Sun, Two scale evolution during shear reversal in dense suspensions, Phys. Rev. E 93, 012604 (2016).

[12] M. D. A. Cooley and M. E. O'Neill, On the slow motion generated in a viscous fluid by the approach of a sphere to a plane wall or stationary sphere, Mathematika 16, 37 (1969).

[13] M. E. O'Neill and S. R. Majumdar, Asymmetrical slow viscous fluid motions caused by the translation or rotation of two spheres. Part II: Asymptotic forms of the solutions when the minimum clearance between the spheres approaches zero, Z. Angew. Math. Phys. 21, 180 (1970).

[14] D. J. Jeffrey and Y. Onishi, Calculation of the resistance and mobility functions for two unequal rigid spheres in low-Reynolds-number flow, J. Fluid Mech. 139, 261 (1984).

[15] D. J. Jeffrey and Y. Onishi, The forces and couples acting on two nearly touching spheres in low-Reynoldsnumber flow, J. App. Math. Phys. (ZAMP) 35, 634 (1984).

[16] S. L. Dance and M. R. Maxey, Incorporation of lubrication effects into the force-coupling method for particulate two-phase flow, J. Comput. Phys. 189, 212 (2003).

[17] K. Sugiyama and F. Takemura, On the lateral migration of a slightly deformed bubble rising near a vertical plane wall, J. Fluid Mech. 662, 209 (2010).

[18] D. J. Benny, Long waves on liquid film, J. Math. Phys. 45, 150 (1966).

[19] S. P. Lin, Finite amplitude side-band stability of a viscous film, J. Fluid Mech. 63, 417 (1974).

[20] O. Reynolds, On the theory of lubrication and its application to Mr. Beuchamp towers experiments, including an experimental determination of the viscosity of olive oil, Philos. Trans. R. Soc. 177, 157 (1886). 
[21] J. Feng and S. Weinbaum, Lubrication theory in highly compressible porous media: The mechanics of skiing, from red cells to humans, J. Fluid Mech. 422, 281 (2000).

[22] T. Gacka, Z. Zhu, R. Crawford, R. Nathan, and Q. Wu, From red cells to soft lubrication, an experimental study of lift generation inside a compressible porous layer, J. Fluid Mech. 818, 5 (2017).

[23] E. Sawaguchi, A. Matsuda, K. Hama, M. Saito, and Y. Tagawa, Droplet levitation over a moving wall with a steady air film, J. Fluid Mech. 862, 261 (2019).

[24] J. Gu, M. Sakaue, S. Takeuchi, and T. Kajishima, An immersed lubrication model for the fluid flow in a narrow gap region, Powder Technol. 329, 445 (2018).

[25] L. G. Leal, Advanced Transport Phenomena: Fluid Mechanics and Convective Transport (Cambridge University Press, Cambridge, UK, 2007).

[26] G. H. Wannier, A contribution to the hydrodynamics of lubrication, Q. Appl. Math. 8, 1 (1950).

[27] S. Takeuchi, H. Fukuoka, J. Gu, and T. Kajishima, Interaction problem between fluid and membrane by a consistent direct discretization approach, J. Comput. Phys. 371, 1018 (2018).

[28] É. Guazzelli and J. F. Morris, A Physical Introduction to Suspension Dynamics (Cambridge University Press, Cambridge, UK, 2011). 